\title{
Hybrid modeling of diffusive shock acceleration in collisionless shocks in multispecies plasma.
}

\author{
J. A. Kropotina ${ }^{1,2 *}$, A. M.Bykov ${ }^{1,2}$, V. E. Ermolina ${ }^{1}$, S. M. Osipov ${ }^{2}$, \\ V. I. Romansky ${ }^{2}$ \\ 1 Peter the Great St.Petersburg Polytechnic University (SPbPU), Russia \\ 2 Ioffe Institute, Russia \\ *E-mail: juliett.k@gmail.com
}

\begin{abstract}
Diffusive shock acceleration (DSA) is a very efficient mechanism of high energy particle acceleration in heliosphere, supernova remnants, stellar winds and gamma-ray bursts. We present microscopic simulation of particle injection and diffusive shock acceleration which is performed with 3D divergence-conserving second-order accurate hybrid code "Maximus". Hydrogen plasma with admixture of various heavy ions is considered. The injection process is found to start through shock reflection for both hydrogen an heavier ions. However, the reflection process depends on charge-to-mass ratio. While hydrogen ions reflection appears at shock ramp and is governed by the cross-shock potential, the reflection of ions with greater $A / Z$ proceeds deeper downstream via gyration in perpendicular magnetic field component. The heavy ions appear to inject into the DSA preferentially, but this chemical enhancement saturates with growing $A / Z$. The protons injection efficiency is estimated within various approaches, and it is shown that about $20 \%$ of initial flow energy goes into accelerated particles.
\end{abstract}

Keywords: Cosmic Rays - Diffusive Shock Acceleration - injection.

\section{Introduction}

The Cosmic Rays (CR) - extremely energetic space particles - are observed both directly in the vicinity of the Earth and via X-ray and gammaray observations in distant sources (See, e.g., Aharonian et al. 2012). The latter observations confirm that the Supernova Remnant (SNR) shocks are effective CR accelerators and can be considered as the major Galactic CR sources. The other astrophysical collisionless shocks can effectively accelerate particles as well. The main mechanism proposed for this process is DSA 
(first order Fermi mechanism), which consists of particles multiple reflection upstream and downstream of the shock. This process leads to constant energy gain of particles and the formation of the power law spectrum with depending on shock compession (Bell 1978).

The details of the DSA process are extensively studied in the last decades (see, e.g., Caprioli \& Spitkovsky 2014, Sundberg et al. 2015), mostly by means of numerical modeling. Particularly, the special attention is payed to the investigation of injection and acceleration of ions heavier than protons (see, e.g., Caprioli et al. 2017). The detailed investigation of this topic can help to resolve the kinetics of heavy ions in collisionless shocks, estimate the fluxes of accelerated heavy ions from supernova shocks and, therefore, better understand the observed anomalies in cosmic rays composition. The existing works (Caprioli et al. 2017, Hanush et al. 2017, 2018) confirm the preferential injection of ions with high mass-to-charge ratio, but do not show consistency in dependency of the injection enhancement on $A / Z$. Also the individual heavy ions trajectories and kinetics were not addressed yet. So in the current paper the results of (Caprioli et al. 2017) are extended to the heavy weakly charged elements, and the saturation of the injection enhancement with growing $A / Z$, in consistency with (Hanush et al 2017, 2018), is shown.

Also the analyses of individual particles trajectories are performed, revealing a slightly different injection mechanism for the ions with high $A / Z$. The multispecies collisionless shock is simulated by means of the $3 \mathrm{~d}$ second order accurate divergence-conserving hybrid code "Maximus" (Kropotina et al. 2018). Such type of codes is well suited for kinetic simulations of ion-governed processes (see, e.g., Winske 1985).

The overall cosmic rays acceleration efficiency, defined as the fraction of bulk kinetic energy going into accelerated particles, is also of special interest. This parameter plays a crucial role, e.g., in simulations of stars and galaxies formation and evolution. So the new technique of CR acceleration efficiency is proposed, revealing nearly $20 \%$ efficiency of bulk kinetic energy conversion to energetic particles.

\section{Simulation}

Collisionless shock simulation was performed with the three-dimensional second-order accurate divergence-conserving hybrid code "Maximus" (Kropotina et al. 2018). The dimensionless quantities, measured in units of the far upstream proton inertial length $\left(l_{i}\right)$, gyrofrequency $\left(\Omega_{i}\right)$, alfvenic $\operatorname{velocity}\left(V_{a}\right)$ and plasma density, were introduced. Shock was initialized via the wall reflection of the super-alfvenic flow moving in negative $\mathrm{x}$ direction (the so-called rigid piston method). The initially uniform magnetic field was inclined by $\theta=10^{\circ}$ to the shock normal (the quasi-parallel configuration is 
believed to be optimal for the effective DSA process (Caprioli \& Spitkovsky 2014)). Shock parameters, characteristic for the supernova remnant forward shocks, were chosen: the initial flow afvenic Mach number $\mathrm{Ma}=10$ (in the downstream frame), the upstream ratio of thermal to magnetic pressure $\beta=1.0$. The hydrogen plasma with negligible admixture of various heavy ions (namely $\mathrm{He}(+2), \mathrm{He}(+1), \mathrm{C}(+3), \mathrm{C}(+2), \mathrm{C}(+1), \mathrm{O}(+1)$ ) was considered. Simulation was performed in 2 d rectangular box of size $40000 \times 1 \times 100$ proton inertial lengths until time $T=2800 \Omega_{i}^{-1}$. So long time ensured that the DSA spectrum completely formed for most ions.

\section{Heavy ions injection enhancement}

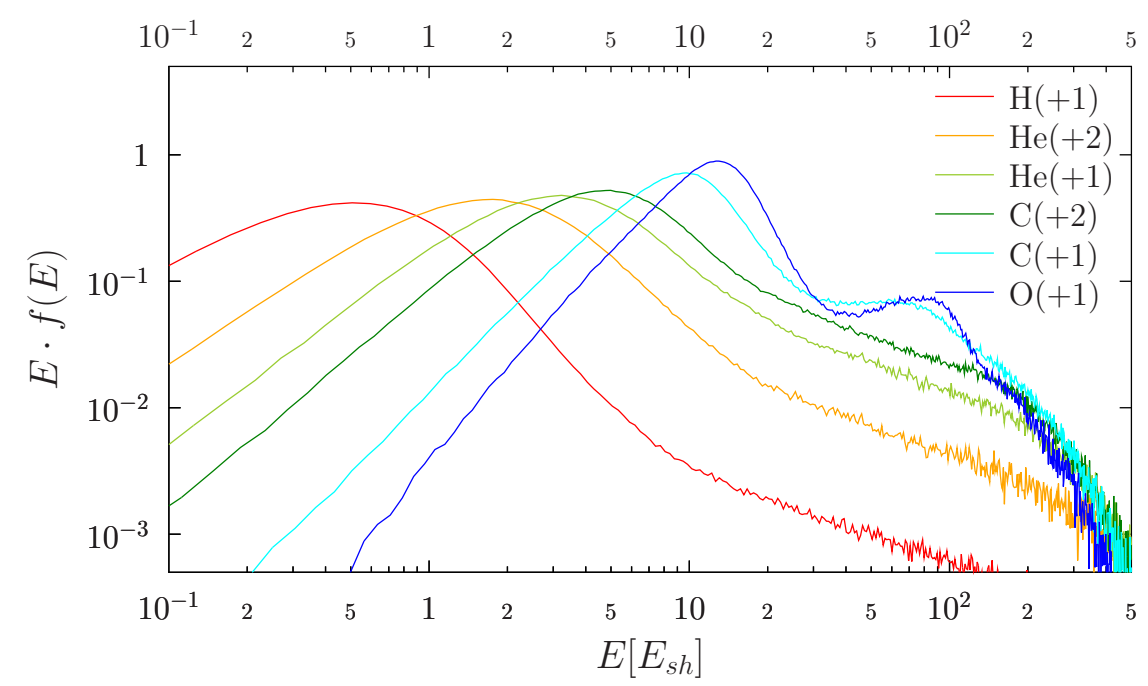

Figure 1: Various ions energy spectra far downstream of the shock with $M_{a}=10, \theta=10^{\circ}, \beta=1$

The resulting far downstream ions spectra are shown in fig. 1. The distribution functions of various sorts are normalized by unit to compare injection efficiency. The maxwellian core and nonthermal power law parts of the spectrum are clearly distinguishable for all sorts. However, it should be noted that the most heavy and weakly charged ions $(\mathrm{C}(+1)$ and $\mathrm{O}(+1))$ still show prominent deviations from both maxwellian distribution in the low-energy part and simple power-law at high energies. This behaviour just reflects the fact that all processes with such ions are much slower than those for the protons, and they have not relaxed to the steady distributions yet. Some ions with smaller $A / Z(\mathrm{C}(+3), \mathrm{C}(+2))$ showed similar features at ear- 
lier times, so $\mathrm{C}(+1)$ and $\mathrm{O}(+1)$ are expected to relax later.

The chemical enhancement (growth of the power law normalization with $A / Z)$ can be seen. This effect was first shown by Caprioli et al. 2017, where authors considered sorts with $A / Z$ up to 8 and proposed linear growth of the enhancement with $A / Z$. At the same time the $1 \mathrm{~d}$ simulations in Hanush et al. 2018 showed the saturation and further decrease of the chemical enhancement for $A / Z>8$. The latter results are confirmed by our two dimensional model.

\section{Ions injection process}

In previous works (Capriloli \& Spitkovsky 2014, Caprioli et al. 2015) the protons injection process was studied by means of individual particle trajectories analyses. The general conclusion was that the injection into the DSA process proceeds via reflection of a fraction of bulk particles during the first shock encounter. After that the reflected ions enter the shock drift acceleration (SDA) process, when they move along the electric field in the shock surface and gain sufficient energy to eventually escape upstream and enter the DSA. In current work the trajectories of accelerated heavy ions were analysed as well, showing that the principal injection mechanism remains the same for all sorts. At the same time, the injection details differ for ions with high $A / Z$.

Fig. 2 shows the phase space trajectories of accelerated particles at times
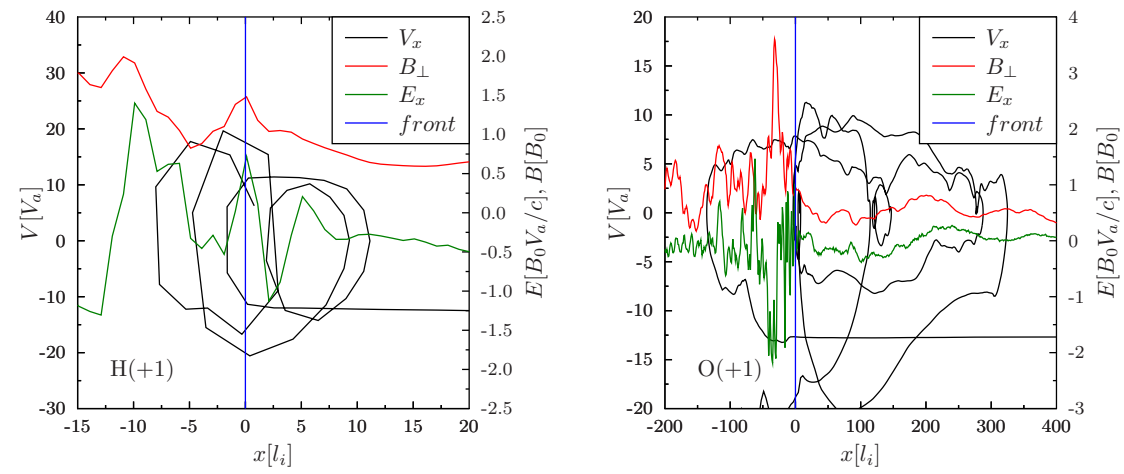

Figure 2: Trajectories of the accelerated protons and $\mathrm{O}(+1)$ ions in the same shock (the corresponding electromagnetic fields are shown for clarity).

close to their first front reflection. The corresponding perpendicular magnetic field and parallel electric field are shown in the same figure (right 
axes). From the left panel it is clear that the reflection point of hydrogen ions coincides with both $B_{\perp}$ and $E_{x}$ maxima. This means that the shock transition is governed by the protons reflection and in turn tuned for it. It is actually hard to distinguish the which reason leads to the protons refection - locally perpendicular magnetic field or cross-shock potential, as they operate together.

\section{Injection efficiency}

The ion injection efficiency can be estimated in various ways. The first one considers just the normalization of the power law tail (Caprioli et al. 2017, Hanush et al. 2017, 2018). However, it does not illustrate the percent of bulk energy going into the accelerated particles. The second way suggests to simply take a ratio of the downstream energy density (or pressure) in the non-thermal part of the spectrum to the total one. This means just an integration of $E \cdot f(E)$ (see fig. 1) from some arbitrary chosen threshold to the maximum energy. However, the downstream distribution function evolves with time, and the power-law tail grows, which means that the estimated injection efficiency grows as well and is limited only by the system size. Moreover, this method does not take into account those accelerated particles which currently reside in the upstream region. In the current paper we suggest a self-consistent way of estimating the injection efficiency from the hybrid simulations. To do it the time evolution of the energy in particles with $E>E_{0}=10 E_{s h}$ and of the total kinetic energy is calculated at each time step. Both appear to grow up nearly linearly after $t \approx 100 \Omega_{i}^{-1}$. This means that their averaged slopes ratio is constant in time and can be treated as the ions injection efficiency. For the considered shock its value corresponded to about 20\%. The same value can be obtained from the approximate downstream temperature, which appears to be about $75-80 \%$ of that expected from the Hugonio relations. This means that the rest of the energy came into magnetic field amplification (which usually takes about a few percent) and CR acceleration.

\section{Summary}

The hybrid code "Maximus" was used to simulate collisionless shocks in hydrogen plasma with the admixture of various heavy ions. The injection process was found to start through the shock reflection for both hydrogen an heavier ions. However, while hydrogen ions reflection appears at shock ramp and is governed by the cross-shock potential, the reflection of ions with greater $A / Z$ proceeds deeper downstream via gyration in perpendicular magnetic field component. The heavy ions appear to inject into the DSA preferentially, but this chemical enhancement saturates with growing $A / Z$. 
The protons injection efficiency is estimated within various approaches, and it is shown that about $20 \%$ of initial flow energy goes into accelerated particles.

\section{Acknowledgements}

The reported study was funded by RFBR according to the research project $18-32-00158$

\section{References}

Aharonian F., Bykov A., Etienne P. et al. 2012, SSR 166, 97

Bell, A. R. 1978, MNRAS 182, 147

Caprioli D., Pop A.-R., Spitkovsky, A. 2015, ApJL 798, L28R

Caprioli D., Spitkovsky A. 2014, ApJ 783, 2, 783:91

Caprioli D., Yi D.T., Spitkovsky A. 2017, Physical Review Letters 119, 171101

Hanusch A., Liseykina T., Malkov M. 2017, 35th International Cosmic Ray Conference, 10-20

July, Bexco, Busan, Korea

Hanusch A., Liseykina T., Malkov M. 2018, ArXiv:1803.00428v1

Kropotina J. A., Bykov A.M., Krassiltchikov A.M., Levenfish K.P. 2018, Communications in

Computer and Information Science, in press Winske D. 1985, SSR 42, 53

Caprioli D., Pop A.-R., Spitkovsky, A. 2015, ApJL 798, L28R

Sundberg T., Haynes C.T., Burgess D., Mazelle C. X. 2015, ApJ 820, 1, 21 